\title{
Nickel-Specific Response in the Transcriptional Regulator, Escherichia coli NikR
}

Sharon Leitch ${ }^{\dagger}$, Michael J. Bradley ${ }^{\ddagger}$, Jessica L. Rowe ${ }^{\ddagger}$, Peter T. Chivers ${ }^{\ddagger}$, and Michael J. Maroney *

University of Massachusetts, Department of Chemistry, Amherst, Massachusetts 01003 and Washington University School of Medicine, Department of Biochemistry and Molecular Biophysics, Saint Louis, Missouri 63110.

mmaroney@chemistry.umass.edu

RECEIVED DATE (to be automatically inserted after your manuscript is accepted if required according to the journal that you are submitting your paper to)

Nickel specific response in NikR

${ }^{\dagger}$ University of Massachusetts ${ }^{\star}$ Washington University 
Table 1. Fourier-Filtered Fits for Co(II)-NikR

\begin{tabular}{|c|c|c|c|c|c|}
\hline \multicolumn{6}{|c|}{$($ Backtransform Window $=1-2.3 \AA)$} \\
\hline & $\mathrm{N}$ & $r(\AA)$ & $\sigma^{2}\left(x 10^{3} \AA^{-2}\right)$ & $\Delta \mathrm{E}_{0}$ & GOF \\
\hline \multirow[t]{2}{*}{ Single shell fits } & $6 \mathrm{Co}-\mathrm{N} / \mathrm{O}$ & $2.11(2)$ & $5.9(12)$ & $7.3(23)$ & 0.77 \\
\hline & $7 \mathrm{Co}-\mathrm{N} / \mathrm{O}$ & $2.10(2)$ & $7.2(13)$ & $7.0(22)$ & 0.75 \\
\hline \multirow[t]{4}{*}{ Two shell fits } & $6 \mathrm{Co}-\mathrm{N} / \mathrm{O}$ & $2.11(2)$ & $5.2(12)$ & $8.2(33)$ & 0.81 \\
\hline & $1 \mathrm{Co}-\mathrm{N} / \mathrm{O}$ & $2.21(8)$ & {$[5.2]$} & {$[8.2]$} & \\
\hline & $5 \mathrm{Co}-\mathrm{N} / \mathrm{O}$ & $2.09(2)$ & $4.5(43)$ & $7.8(40)$ & 0.85 \\
\hline & $1 \mathrm{Co}-\mathrm{N} / \mathrm{O}$ & $2.20(20)$ & {$[4.5]$} & {$[7.8]$} & \\
\hline \multicolumn{6}{|c|}{ Backtransform Window $=1-4 \AA$} \\
\hline & $6 \mathrm{Co}-\mathrm{N} / \mathrm{O}$ & $2.11(2)$ & $6.1(13)$ & $8.2(22)$ & 1.20 \\
\hline & $7 \mathrm{Co}-\mathrm{N} / \mathrm{O}$ & $2.11(2)$ & $7.4(13)$ & $7.9(21)$ & 1.19 \\
\hline \multirow[t]{6}{*}{ Imidazole fits } & $2 \mathrm{Co}-\mathrm{N}_{\text {imd }}$ & $2.06(5)$ & $5.0(27)$ & $6.9(21)$ & 0.78 \\
\hline & $4 \mathrm{Co}-\mathrm{N} / \mathrm{O}$ & $2.12(2)$ & {$[5.0]$} & [6.9] & \\
\hline & $4 \mathrm{Co}-\mathrm{C}_{\mathrm{imd}}$ & $2.95(3)$ & $4.1(25)$ & $9.8(36)$ & \\
\hline & $3 \mathrm{Co}-\mathrm{N}_{\mathrm{imd}}$ & $2.05(1)$ & $0.8(16)$ & $6.6(16)$ & 0.65 \\
\hline & $3 \mathrm{Co}-\mathrm{N} / \mathrm{O}$ & $2.17(1)$ & {$[0.8]$} & [6.6] & \\
\hline & $6 \mathrm{Co}-\mathrm{C}_{\mathrm{imd}}$ & $2.96(2)$ & $5.9(2)$ & $10.2(21)$ & \\
\hline
\end{tabular}




\begin{tabular}{|c|c|c|c|c|}
\hline $4 \mathrm{Co}-\mathrm{N}_{\mathrm{imd}}$ & $2.09(1)$ & $3.9(21)$ & $9.0(15)$ & 0.73 \\
\hline $2 \mathrm{Co}-\mathrm{N} / \mathrm{O}$ & $2.19(4)$ & [3.9] & [9.0] & \\
\hline $8 \mathrm{Co}-\mathrm{C}_{\mathrm{imd}}$ & $2.93(3)$ & $8.4(23)$ & $6.5(23)$ & \\
\hline $2 \mathrm{Co}-\mathrm{N}_{\mathrm{imd}}$ & $2.04(5)$ & $6.0(30)$ & $6.2(19)$ & 0.77 \\
\hline 5 Co-N/O & $2.12(2)$ & [6.0] & [6.2] & \\
\hline $4 \mathrm{Co}-\mathrm{C}_{\mathrm{imd}}$ & $2.95(3)$ & $4.7(24)$ & $10.7(33)$ & \\
\hline $3 \mathrm{Co}^{-\mathrm{N}_{\text {imd }}}$ & $2.04(2)$ & $3.7(26)$ & $5.3(16)$ & 0.67 \\
\hline $4 \mathrm{Co}-\mathrm{N} / \mathrm{O}$ & $2.14(1)$ & [3.7] & [5.3] & \\
\hline $6 \mathrm{Co}-\mathrm{C}_{\mathrm{imd}}$ & $2.96(2)$ & $6.3(21)$ & $11.1(20)$ & \\
\hline $4 \mathrm{Co}-\mathrm{N}_{\text {imd }}$ & $2.07(1)$ & $4.0(2)$ & $8.5(16)$ & 0.74 \\
\hline $3 \mathrm{Co}-\mathrm{N} / \mathrm{O}$ & $2.18(3)$ & {$[4.0]$} & {$[8.5]$} & \\
\hline $8 \mathrm{Co}-\mathrm{C}_{\mathrm{imd}}$ & $2.93(3)$ & $8.2(2)$ & $6.7(24)$ & \\
\hline
\end{tabular}


Table 2. Fourier-Filtered Fits for $\mathrm{Cu}(\mathrm{II})-\mathrm{NikR}$

\begin{tabular}{|c|c|c|c|c|c|}
\hline \multicolumn{6}{|c|}{ Backtransform Window $=1-2.3 \AA$} \\
\hline & $\mathrm{N}$ & $\mathrm{r}(\AA)$ & $\sigma^{2}\left(x 10^{3} \AA^{-2}\right)$ & $\Delta \mathrm{E}_{0}$ & GOF \\
\hline \multirow[t]{2}{*}{ Single shell fits } & $4 \mathrm{Cu}-\mathrm{N} / \mathrm{O}$ & $2.00(3)$ & $9.5(32)$ & $11.4(39)$ & 0.96 \\
\hline & $4 \mathrm{Cu}-\mathrm{S}$ & $2.13(3)$ & $17.3(21)$ & $-7.5(33)$ & 0.58 \\
\hline \multirow[t]{5}{*}{ Two shell fits } & $3 \mathrm{Cu}-\mathrm{N} / \mathrm{O}$ & $1.93(2)$ & $9.8(11)$ & $0.6(27)$ & 0.22 \\
\hline & $1 \mathrm{Cu}-\mathrm{S}$ & $2.23(1)$ & $4.7(8)$ & $9.6(23)$ & \\
\hline & $2 \mathrm{Cu}-\mathrm{N} / \mathrm{O}$ & $1.91(2)$ & $5.1(17)$ & $2.8(27)$ & 0.20 \\
\hline & $1 \mathrm{Cu}-\mathrm{N} / \mathrm{O}$ & $2.06(5)$ & {$[5.1]$} & {$[2.8]$} & \\
\hline & $1 \mathrm{Cu}-\mathrm{S}$ & $2.22(1)$ & $4.6(8)$ & $9.2(21)$ & \\
\hline \multicolumn{6}{|c|}{ Backtransform Window $=1-4 \AA$} \\
\hline & $2 \mathrm{Cu}-\mathrm{N} / \mathrm{O}$ & $1.91(4)$ & $4.5(28)$ & $3.2(52)$ & 0.72 \\
\hline & $1 \mathrm{Cu}-\mathrm{N} / \mathrm{O}$ & $2.07(11)$ & {$[4.5]$} & {$[3.2]$} & \\
\hline & $1 \mathrm{Cu}-\mathrm{S}$ & $2.21(3)$ & $4.1(22)$ & $8.1(48)$ & \\
\hline \multirow[t]{3}{*}{ Imidazole fits } & $2 \mathrm{Cu}-\mathrm{N}_{\text {imd }}$ & $1.92(1)$ & $4.1(8)$ & $4.5(13)$ & 0.35 \\
\hline & $1 \mathrm{Cu}-\mathrm{N} / \mathrm{O}$ & $2.10(4)$ & [4.1] & {$[4.5]$} & \\
\hline & $1 \mathrm{Cu}-\mathrm{S}$ & $2.21(1)$ & $3.8(13)$ & $7.4(25)$ & \\
\hline
\end{tabular}


Table 3. Fourier-Filtered Fits for $\mathrm{Cu}(\mathrm{I})-\mathrm{NikR}$

\begin{tabular}{|c|c|c|c|c|c|}
\hline \multicolumn{6}{|c|}{ Backtransform Window $=1-2.3 \AA$} \\
\hline & $\mathrm{N}$ & $\mathrm{r}(\AA)$ & $\sigma^{2}\left(x 10^{3} \AA^{-2}\right)$ & $\Delta \mathrm{E}_{0}$ & GOF \\
\hline \multirow[t]{3}{*}{ Single shell fits } & $3 \mathrm{Cu}-\mathrm{N} / \mathrm{O}$ & $2.16(1)$ & $0.6(6)$ & $24.1(16)$ & 0.67 \\
\hline & $2 \mathrm{Cu}-\mathrm{S}$ & $2.25(1)$ & $2.9(6)$ & $2.3(23)$ & 0.63 \\
\hline & $3 \mathrm{Cu}-\mathrm{S}$ & $2.25(1)$ & $5.4(8)$ & $1.6(23)$ & 0.72 \\
\hline \multirow[t]{2}{*}{ Two shell fits } & $2 \mathrm{Cu}-\mathrm{N} / \mathrm{O}$ & $2.07(2)$ & $2.3(31)$ & $19.8(23)$ & 0.44 \\
\hline & $1 \mathrm{Cu}-\mathrm{S}$ & $2.26(1)$ & $-0.9(11)$ & $-4.1(46)$ & \\
\hline \multicolumn{6}{|c|}{ Backtransform Window $=1-4.0 \AA$} \\
\hline & $2 \mathrm{Cu}-\mathrm{N} / \mathrm{O}$ & $2.09(5)$ & $5.6(40)$ & $15.7(43)$ & 1.07 \\
\hline & $1 \mathrm{Cu}-\mathrm{S}$ & $2.28(2)$ & $0.2(10)$ & $7.1(58)$ & \\
\hline \multirow[t]{5}{*}{ Imidazole fits } & $2 \mathrm{Cu}-\mathrm{N}_{\text {imd }}$ & $2.08(4)$ & $5.9(33)$ & $11.5(36)$ & 1.06 \\
\hline & $1 \mathrm{Cu}-\mathrm{S}$ & $2.28(2)$ & $0.1(8)$ & $8.1(45)$ & \\
\hline & $1 \mathrm{Cu}-\mathrm{N}_{\text {imd }}$ & $2.14(6)$ & $2.7(47)$ & $14.1(42)$ & 1.05 \\
\hline & $1 \mathrm{Cu}-\mathrm{N} / \mathrm{O}$ & $2.04(5)$ & {$[2.7]$} & {$[14.1]$} & \\
\hline & $1 \mathrm{Cu}-\mathrm{S}$ & $2.28(2)$ & $0.1(8)$ & $7.1(50)$ & \\
\hline
\end{tabular}


Table 4. Fourier-Filtered Fits for Zn(II)-NikR

\begin{tabular}{|c|c|c|c|c|c|}
\hline \multicolumn{6}{|c|}{ Backtransform Window $=1-2.3 \AA$} \\
\hline & $\mathrm{N}$ & $\mathrm{r}(\AA)$ & $\sigma^{2}\left(\times 10^{3} \AA^{-2}\right)$ & $\Delta \mathrm{E}_{0}$ & GOF \\
\hline \multirow[t]{2}{*}{ Single shell fits } & $4 \mathrm{Zn}-\mathrm{N} / \mathrm{O}$ & $2.05(2)$ & $4.9(17)$ & $10.3(32)$ & 0.98 \\
\hline & $4 \mathrm{Zn}-\mathrm{S}$ & $2.16(1)$ & $10.1(9)$ & $-7.2(25)$ & 0.45 \\
\hline \multirow[t]{4}{*}{ Two shell fits } & $3 \mathrm{Zn}-\mathrm{N} / \mathrm{O}$ & $2.00(2)$ & $2.9(1)$ & $4.1(31)$ & 0.53 \\
\hline & $1 \mathrm{Zn}-\mathrm{S}$ & $2.28(3)$ & $2.9(16)$ & $9.4(54)$ & \\
\hline & $4 \mathrm{Zn}-\mathrm{N} / \mathrm{O}$ & $2.02(1)$ & $5.2(9)$ & $5.0(22)$ & 0.38 \\
\hline & $1 \mathrm{Zn}-\mathrm{S}$ & $2.31(3)$ & $5.1(16)$ & $16.8(44)$ & \\
\hline \multicolumn{6}{|c|}{ Backtransform Window $=1-4.0 \AA$} \\
\hline & $3 \mathrm{Zn}-\mathrm{N} / \mathrm{O}$ & $2.00(2)$ & $3.2(16)$ & $4.3(39)$ & 1.03 \\
\hline & $1 \mathrm{Zn}-\mathrm{S}$ & $2.26(3)$ & $3.0(21)$ & $6.4(75)$ & \\
\hline & $4 \mathrm{Zn}-\mathrm{N} / \mathrm{O}$ & $2.04(2)$ & $5.0(1)$ & $8.7(24)$ & 0.94 \\
\hline & $1 \mathrm{Zn}-\mathrm{S}$ & $2.37(4)$ & $7.6(3)$ & $28.7(51)$ & \\
\hline & $3 \mathrm{Zn}-\mathrm{N}_{\mathrm{imd}}$ & $1.99(2)$ & $4.7(12)$ & $3.1(18)$ & 0.75 \\
\hline & $1 \mathrm{Zn}-\mathrm{S}$ & $2.24(2)$ & $3.7(15)$ & $4.2(55)$ & \\
\hline & $3 \mathrm{Zn}-\mathrm{N}_{\text {imd }}$ & $1.99(2)$ & $5.8(16)$ & $3.5(18)$ & 0.68 \\
\hline
\end{tabular}




$\begin{array}{llll}1 \mathrm{Zn}-\mathrm{N} / \mathrm{O} & 2.04(7) & {[5.8]} & {[3.5]} \\ 1 \mathrm{Zn}-\mathrm{S} & 2.28(3) & 5.3(18) & 11.1(48)\end{array}$


Table 5. Fourier-Filtered Fits for CuNi-NikR (Cu-site)

\begin{tabular}{|c|c|c|c|c|c|}
\hline \multicolumn{6}{|c|}{ Backtransform Window $=1-2.3 \AA$} \\
\hline & $\mathrm{N}$ & $\mathrm{r}(\AA)$ & $\sigma^{2}\left(x 10^{3} \AA^{-2}\right)$ & $\Delta \mathrm{E}_{0}$ & GOF \\
\hline \multirow[t]{2}{*}{ Single shell fits } & $4 \mathrm{Cu}-\mathrm{N} / \mathrm{O}$ & $1.96(3)$ & $9.7(26)$ & $7.0(37)$ & 0.78 \\
\hline & $4 \mathrm{Cu}-\mathrm{S}$ & $2.08(3)$ & $16.4(22)$ & $-12.7(50)$ & 0.64 \\
\hline \multirow[t]{5}{*}{ Two shell fits } & $3 \mathrm{Cu}-\mathrm{N} / \mathrm{O}$ & $1.98(16)$ & $6.7(13)$ & $8.7(36)$ & \\
\hline & $1 \mathrm{Cu}-\mathrm{S}$ & $2.20(13)$ & $24.6(140)$ & $19.0(140)$ & \\
\hline & $2 \mathrm{Cu}-\mathrm{N} / \mathrm{O}$ & $1.98(2)$ & $2.4(17)$ & $10.6(26)$ & 0.23 \\
\hline & $1 \mathrm{Cu}-\mathrm{N} / \mathrm{O}$ & $2.14(7)$ & [2.4] & {$[10.6]$} & \\
\hline & $1 \mathrm{Cu}-\mathrm{S}$ & $2.19(4)$ & $14.0(120)$ & $15.8(80)$ & \\
\hline \multicolumn{6}{|c|}{ Backtransform Window $=1-4.0 \AA$} \\
\hline & $2 \mathrm{Cu}-\mathrm{N} / \mathrm{O}$ & $1.98(2)$ & $2.5(22)$ & $10.7(25)$ & 0.46 \\
\hline & $1 \mathrm{Cu}-\mathrm{N} / \mathrm{O}$ & $2.14(9)$ & {$[2.5]$} & {$[10.7]$} & \\
\hline & $1 \mathrm{Cu}-\mathrm{S}$ & $2.18(6)$ & $13.4(12)$ & $14.1(59)$ & \\
\hline \multirow[t]{4}{*}{ Imidazole fits } & $2 \mathrm{Cu}-\mathrm{N}_{\mathrm{imd}}$ & $1.97(3)$ & $6.7(12)$ & $8.3(21)$ & 0.39 \\
\hline & $1 \mathrm{Cu}-\mathrm{N} / \mathrm{O}$ & $2.01(5)$ & {$[6.7]$} & [8.3] & \\
\hline & $1 \mathrm{Cu}-\mathrm{S}$ & $2.17(7)$ & $23.8(110)$ & $14.9(73)$ & \\
\hline & $1 \mathrm{Cu}-\mathrm{N}_{\mathrm{imd}}$ & $1.95(1)$ & $5.8(13)$ & $8.2(25)$ & 0.37 \\
\hline
\end{tabular}




$\begin{array}{llll}2 \mathrm{Cu}-\mathrm{N} / \mathrm{O} & 2.00(2) & {[5.8]} & {[8.2]} \\ 1 \mathrm{Cu}-\mathrm{S} & 2.15(6) & 24.8(110) & 14.1(70)\end{array}$

Table 6. Fourier-Filtered Fits for CuNi-NikR (Ni-site)

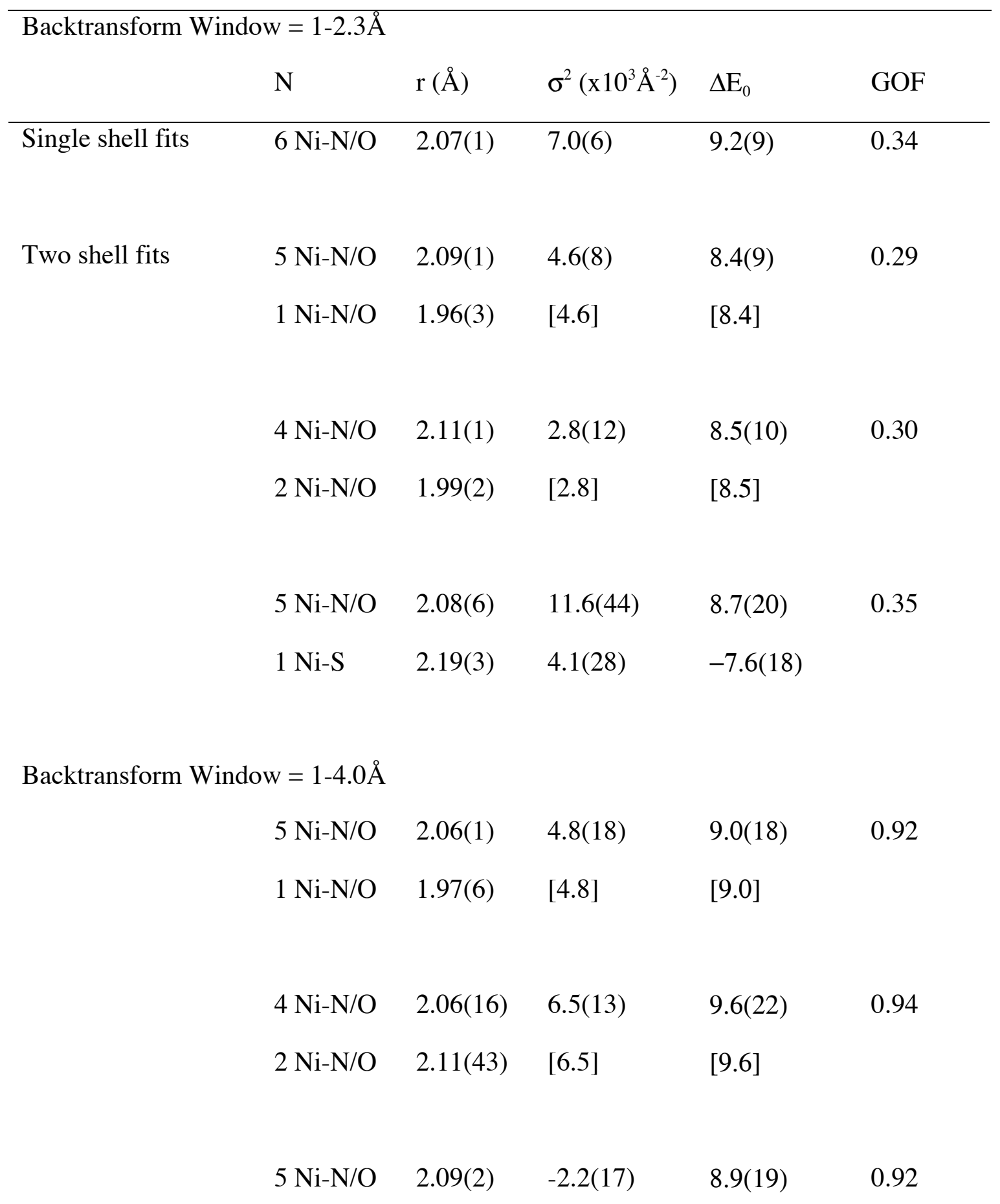


$1 \mathrm{Ni}-\mathrm{S} \quad 2.23(2) \quad-4.5(17) \quad-55.2(7.2)$

Imidazole fits

$\begin{array}{lllll}2 \mathrm{Ni}-\mathrm{N}_{\text {imd }} & 2.01(2) & 4.0(1) & 10.1(10) & 0.65 \\ 4 \mathrm{Ni}-\mathrm{N} / \mathrm{O} & 2.22(1) & {[4.0]} & {[10.1]} & \\ & & & & \\ & & & & \\ 1 \mathrm{Ni}^{-N_{\text {imd }}} & 1.99(5) & 5.3(15) & 9.7(14) & 0.78 \\ 5 \mathrm{Ni}-\mathrm{N} / \mathrm{O} & 2.09(9) & {[5.3]} & {[9.7]} & \end{array}$

$\begin{array}{lllll}3 \mathrm{Ni}^{-} \mathrm{N}_{\mathrm{imd}} & 2.04(1) & 4.1(15) & 11.0(9) & 0.64 \\ 3 \mathrm{Ni}-\mathrm{N} / \mathrm{O} & 2.14(1) & {[4.1]} & {[11.0]} & \end{array}$

$\begin{array}{lllll}2 \mathrm{Ni}-\mathrm{N}_{\text {imd }} & 2.03(2) & 2.5(14) & 11.1(12) & 0.65 \\ 3 \mathrm{Ni}-\mathrm{N} / \mathrm{O} & 2.13(1) & {[2.5]} & {[11.1]} & \\ 1 \mathrm{Ni}-\mathrm{S} / \mathrm{Cl} & 1.94(27) & 17.2(12) & -49.0(67) & \end{array}$

$\begin{array}{lllll}1 \mathrm{Ni}-\mathrm{N}_{\text {imd }} & 2.02(5) & 4.0(17) & 10.7(18) & 0.82 \\ 4 \mathrm{Ni}-\mathrm{N} / \mathrm{O} & 2.10(1) & {[4.0]} & {[10.7]} & \\ 1 \mathrm{Ni}-\mathrm{S} / \mathrm{Cl} & 1.97(34) & 16.8(130) & -41.3(780) & \end{array}$

$\begin{array}{lllll}3 \mathrm{Ni}-\mathrm{N}_{\text {imd }} & 2.04(2) & 4.7(23) & 12.1(13) & 0.65 \\ 2 \mathrm{Ni}-\mathrm{N} / \mathrm{O} & 2.20(6) & {[4.7]} & {[12.1]} & \\ 1 \mathrm{Ni}-\mathrm{S} / \mathrm{Cl} & 2.19(4) & 2.2(22) & -12.3(110) & \end{array}$


Table 7. Fourier-Filtered Fits for NiCo-NikR (Ni-site)

\begin{tabular}{|c|c|c|c|c|c|}
\hline \multicolumn{6}{|c|}{ Backtransform Window $=1-2.3 \AA$} \\
\hline & $\mathrm{N}$ & $r(\AA)$ & $\sigma^{2}\left(x 10^{3} \AA^{-2}\right)$ & $\Delta \mathrm{E}_{0}$ & GOF \\
\hline \multirow[t]{2}{*}{ Single shell fits } & $4 \mathrm{Ni}-\mathrm{N} / \mathrm{O}$ & $1.97(4)$ & $13.5(37)$ & $8.6(40)$ & 0.74 \\
\hline & $4 \mathrm{Ni}-\mathrm{S}$ & $2.10(3)$ & $17.9(23)$ & $-8.0(48)$ & 0.57 \\
\hline \multirow[t]{5}{*}{ Two shell fits } & $3 \mathrm{Ni}-\mathrm{N} / \mathrm{O}$ & $1.88(7)$ & $16.5(55)$ & $-1.3(78)$ & 0.49 \\
\hline & $1 \mathrm{Ni}-\mathrm{S}$ & $2.18(3)$ & $5.9(22)$ & $3.2(78)$ & \\
\hline & $2 \mathrm{Ni}-\mathrm{N} / \mathrm{O}$ & $1.93(4)$ & $5.6(37)$ & $9.5(71)$ & 0.40 \\
\hline & $1 \mathrm{Ni}-\mathrm{N} / \mathrm{O}$ & $2.32(9)$ & {$[5.6]$} & [9.5] & \\
\hline & $1 \mathrm{Ni}-\mathrm{S}$ & $2.19(2)$ & $3.3(24)$ & $-4.2(88)$ & \\
\hline \multicolumn{6}{|c|}{ Backtransform Window $=1-4.0 \AA$} \\
\hline & $2 \mathrm{Ni}-\mathrm{N} / \mathrm{O}$ & $1.93(4)$ & $4.5(39)$ & $10.9(68)$ & 0.75 \\
\hline & $1 \mathrm{Ni}-\mathrm{N} / \mathrm{O}$ & $2.30(9)$ & [4.5] & [10.9] & \\
\hline & $1 \mathrm{Ni}-\mathrm{S}$ & $2.19(2)$ & $2.2(26)$ & $-3.9(75)$ & \\
\hline \multirow[t]{3}{*}{ Imidazole fits } & $2 \mathrm{Ni}-\mathrm{N}_{\text {imd }}$ & $1.89(1)$ & $0.4(6)$ & $10.0(15)$ & 0.59 \\
\hline & $1 \mathrm{Ni}-\mathrm{N} / \mathrm{O}$ & $2.12(8)$ & {$[0.4]$} & [10.0] & \\
\hline & $1 \mathrm{Ni}-\mathrm{S}$ & $2.16(2)$ & $1.4(36)$ & $7.0(37)$ & \\
\hline
\end{tabular}


Table 8. Fourier-Filtered Fits for NiCo-NikR (Co-site)

\begin{tabular}{|c|c|c|c|c|c|}
\hline \multicolumn{6}{|c|}{ Backtransform Window $=1-2.3 \AA$} \\
\hline & $\mathrm{N}$ & $\mathrm{r}(\AA)$ & $\sigma^{2}\left(x 10^{3} \AA^{-2}\right)$ & $\Delta \mathrm{E}_{0}$ & GOF \\
\hline Single shell fits & $6 \mathrm{Co}-\mathrm{N} / \mathrm{O}$ & $2.04(3)$ & $17.6(25)$ & $-3.4(27)$ & 0.44 \\
\hline \multirow[t]{4}{*}{ Two shell fits } & $3 \mathrm{Co}-\mathrm{N} / \mathrm{O}$ & $1.95(3)$ & $7.0(27)$ & $-4.9(29)$ & 0.43 \\
\hline & $3 \mathrm{Co}-\mathrm{N} / \mathrm{O}$ & $2.12(3)$ & {$[7.0]$} & {$[-4.9]$} & \\
\hline & $5 \mathrm{Co}-\mathrm{N} / \mathrm{O}$ & $1.96(2)$ & $11.5(31)$ & $-7.9(28)$ & 0.29 \\
\hline & $1 \mathrm{Co}-\mathrm{S} / \mathrm{Cl}$ & $2.21(3)$ & $4.0(20)$ & $-28.9(98)$ & \\
\hline \multicolumn{6}{|c|}{ Backtransform Window $=1-4.0 \AA$} \\
\hline & $5 \mathrm{Co}-\mathrm{N} / \mathrm{O}$ & $1.96(4)$ & $12.2(55)$ & $-7.7(48)$ & 0.77 \\
\hline & $1 \mathrm{Co}-\mathrm{S} / \mathrm{Cl}$ & $2.22(4)$ & $4.5(35)$ & $-26.6(17)$ & \\
\hline \multirow[t]{7}{*}{ Imidazole fits } & $2 \mathrm{Co}^{-\mathrm{N}_{\text {imd }}}$ & $1.92(1)$ & $5.8(21)$ & $-6.0(29)$ & 0.53 \\
\hline & $3 \mathrm{Co}-\mathrm{N} / \mathrm{O}$ & $2.12(7)$ & {$[5.8]$} & {$[-6.0]$} & \\
\hline & $1 \mathrm{Co}-\mathrm{S} / \mathrm{Cl}$ & $2.15(6)$ & $9.0(15)$ & $-15.5(120)$ & \\
\hline & $1 \mathrm{Co}-\mathrm{N}_{\text {imd }}$ & & & & No Fit* \\
\hline & $4 \mathrm{Co}-\mathrm{N} / \mathrm{O}$ & & & & \\
\hline & $1 \mathrm{Co}-\mathrm{S} / \mathrm{Cl}$ & & & & \\
\hline & $3 \mathrm{Co}^{-\mathrm{N}_{\text {imd }}}$ & & & & No Fit \\
\hline
\end{tabular}


2 Co-N/O

$1 \mathrm{Co}-\mathrm{S} / \mathrm{Cl}$

"Parameters yielded no refinement. 
Table 9. Fourier-Filtered Fits for NiCo-NikR in TrisBr/NaBr (Ni-site)

\begin{tabular}{|c|c|c|c|c|c|}
\hline \multicolumn{6}{|c|}{ Backtransform Window $=1-2.3 \AA$} \\
\hline & $\mathrm{N}$ & $r(\AA)$ & $\sigma^{2}\left(x 10^{3} \AA^{-2}\right)$ & $\Delta \mathrm{E}_{0}$ & GOF \\
\hline \multirow[t]{2}{*}{ Single shell fits } & $4 \mathrm{Ni}-\mathrm{N} / \mathrm{O}$ & $1.91(3)$ & $8.9(30)$ & $8.4(48)$ & 1.08 \\
\hline & $4 \mathrm{Ni}-\mathrm{S}$ & $2.05(4)$ & $14.3(22)$ & $-8.6(57)$ & 0.85 \\
\hline \multirow[t]{7}{*}{ Two shell fits } & $2 \mathrm{Ni}-\mathrm{N} / \mathrm{O}$ & $1.86(1)$ & $1.8(8)$ & $2.2(32)$ & 0.40 \\
\hline & $1 \mathrm{Ni}-\mathrm{S}$ & $2.17(2)$ & $3.3(10)$ & $8.1(38)$ & \\
\hline & $3 \mathrm{Ni}-\mathrm{N} / \mathrm{O}$ & $1.87(2)$ & $5.2(11)$ & $1.1(32)$ & 0.46 \\
\hline & $1 \mathrm{Ni}-\mathrm{S}$ & $2.21(2)$ & $4.5(13)$ & $14.9(41)$ & \\
\hline & $2 \mathrm{Ni}-\mathrm{N} / \mathrm{O}$ & $1.87(2)$ & $0.9(9)$ & $5.7(33)$ & 0.44 \\
\hline & $1 \mathrm{Ni}-\mathrm{N} / \mathrm{O}$ & $2.02(5)$ & [0.9] & {$[5.7]$} & \\
\hline & $1 \mathrm{Ni}-\mathrm{S}$ & $2.19(2)$ & $5.9(15)$ & $15.2(8)$ & \\
\hline \multicolumn{6}{|c|}{ Backtransform Window $=1-4.0 \AA$} \\
\hline & $2 \mathrm{Ni}-\mathrm{N} / \mathrm{O}$ & $1.88(3)$ & $0.7(16)$ & $6.8(47)$ & 1.19 \\
\hline & $1 \mathrm{Ni}-\mathrm{N} / \mathrm{O}$ & $2.06(11)$ & {$[0.7]$} & {$[6.8]$} & \\
\hline & $1 \mathrm{Ni}-\mathrm{S}$ & $2.17(6)$ & $4.5(61)$ & $11.2(97)$ & \\
\hline \multirow[t]{2}{*}{ Imidazole fits } & $2 \mathrm{Ni}-\mathrm{N}_{\mathrm{imd}}$ & $1.89(9)$ & $0.4(6)$ & $10.0(15)$ & 0.58 \\
\hline & $1 \mathrm{Ni}-\mathrm{N} / \mathrm{O}$ & $2.12(8)$ & {$[0.4]$} & [10.0] & \\
\hline
\end{tabular}


$1 \mathrm{Ni}-\mathrm{S} \quad 2.16(2) \quad 1.4(36) \quad 7.0(37)$ 
Table 10. Fourier-Filtered Fits for NiCo-NikR in TrisBr/NaBr (Co-site)

\begin{tabular}{|c|c|c|c|c|c|}
\hline \multicolumn{6}{|c|}{ Backtransform Window $=1-2.3 \AA$} \\
\hline & $\mathrm{N}$ & $\mathrm{r}(\AA)$ & $\sigma^{2}\left(x 10^{3} \AA^{-2}\right)$ & $\Delta \mathrm{E}_{0}$ & GOF \\
\hline Single shell fits & $6 \mathrm{Co}-\mathrm{N} / \mathrm{O}$ & $2.04(3)$ & $9.1(24)$ & $-0.7(37)$ & 0.71 \\
\hline \multirow[t]{7}{*}{ Two shell fits } & $3 \mathrm{Co}-\mathrm{N} / \mathrm{O}$ & $2.07(2)$ & $1.4(14)$ & $-4.9(25)$ & 0.48 \\
\hline & $2 \mathrm{Co}-\mathrm{N} / \mathrm{O}$ & $1.91(2)$ & & & \\
\hline & $5 \mathrm{Co}-\mathrm{N} / \mathrm{O}$ & $1.92(4)$ & $15.7(38)$ & $-13.1(61)$ & 0.50 \\
\hline & $1 \mathrm{Co}-\mathrm{S} / \mathrm{Cl}$ & $2.21(3)$ & $4.3(20)$ & $-11.5(97)$ & \\
\hline & $3 \mathrm{Co}-\mathrm{N} / \mathrm{O}$ & $2.03(5100)$ & $14.0(230)$ & $-2.5(57)$ & 0.51 \\
\hline & $2 \mathrm{Co}-\mathrm{N} / \mathrm{O}$ & $2.29(7600)$ & {$[14.0]$} & {$[-2.5]$} & \\
\hline & $1 \mathrm{Co}-\mathrm{Br}$ & $2.37(11)$ & $11.1(40)$ & $5.7(260)$ & \\
\hline \multicolumn{6}{|c|}{ Backtransform Window $=1-4.0 \AA$} \\
\hline & $3 \mathrm{Co}-\mathrm{N} / \mathrm{O}$ & $2.08(2)$ & $2.0(19)$ & $-4.1(33)$ & 0.98 \\
\hline & $2 \mathrm{Co}-\mathrm{N} / \mathrm{O}$ & $1.92(3)$ & {$[2.0]$} & {$[-4.1]$} & \\
\hline \multirow[t]{4}{*}{ Imidazole fits } & $2 \mathrm{Co}-\mathrm{N}_{\mathrm{imd}}$ & $1.93(2)$ & $2.2(16)$ & $-2.6(26)$ & 0.80 \\
\hline & $3 \mathrm{Co}-\mathrm{N} / \mathrm{O}$ & $2.09(2)$ & {$[2.2]$} & {$[-2.6]$} & \\
\hline & $4 \mathrm{Co}-\mathrm{C}_{\text {imd }}$ & $3.09(4)$ & $3.9(25)$ & $0.8(40)$ & \\
\hline & $1 \mathrm{Co}-\mathrm{N}_{\text {imd }}$ & $1.89(6)$ & $6.4(21)$ & $-1.9(310)$ & 0.88 \\
\hline
\end{tabular}




\begin{tabular}{|c|c|c|c|}
\hline $4 \mathrm{Co}-\mathrm{N} / \mathrm{O}$ & $2.06(3)$ & [6.4] & [-1.9] \\
\hline $2 \mathrm{Co}^{-\mathrm{C}_{\text {imd }}}$ & $3.07(4)$ & $-0.8(22)$ & $-0.2(670)$ \\
\hline $3 \mathrm{Co}-\mathrm{N}_{\mathrm{imd}}$ & $2.01(3)$ & $8.5(69)$ & $1.8(26)$ \\
\hline $2 \mathrm{Co}-\mathrm{N} / \mathrm{O}$ & $2.13(9)$ & [8.5] & [1.8] \\
\hline $6 \mathrm{Co}-\mathrm{C}_{\mathrm{imd}}$ & $3.05(4)$ & $6.7(33)$ & $-3.4(41)$ \\
\hline
\end{tabular}


Table 12. Unfiltered Fits for High-Affinity Metal Substituted NikR Complexes

\begin{tabular}{|c|c|c|c|c|c|}
\hline & $\mathrm{N}$ & $\mathrm{r}(\AA)$ & $\sigma^{2}\left(x 10^{3} \AA^{-2}\right)$ & $\Delta \mathrm{E}_{0}$ & GOF \\
\hline \multirow{3}{*}{$\mathrm{Co}(\mathrm{II})-\mathrm{NikR}$} & $2 \mathrm{Co}-\mathrm{N}_{\text {imd }}$ & $2.041(20)$ & $3.8(11)$ & $6.5(8)$ & \multirow[t]{3}{*}{1.02} \\
\hline & $4 \mathrm{Co}-\mathrm{N}$ & $2.131(6)$ & [3.8] & {$[6.5]$} & \\
\hline & $4 \mathrm{Co}-\mathrm{C}_{\mathrm{imd}}$ & $2.948(12)$ & $3.9(10)$ & $9.8(15)$ & \\
\hline & $3 \mathrm{Co}-\mathrm{N}_{\text {imd }}$ & $2.047(4)$ & $0.6(6)$ & $6.7(7)$ & \multirow[t]{3}{*}{0.95} \\
\hline & $3 \mathrm{Co}-\mathrm{N}$ & $2.171(6)$ & {$[0.6]$} & {$[6.7]$} & \\
\hline & $6 \mathrm{Co}-\mathrm{C}_{\mathrm{imd}}$ & $2.959(10)$ & $5.8(8)$ & $10.0(10)$ & \\
\hline & $4 \mathrm{Co}-\mathrm{N}_{\mathrm{imd}}$ & $2.084(5)$ & $4.0(9)$ & $8.4(7)$ & 1.00 \\
\hline & $2 \mathrm{Co}-\mathrm{N}$ & $2.179(16)$ & {$[4.0]$} & {$[8.4]$} & \\
\hline & $8 \mathrm{Co}-\mathrm{C}_{\mathrm{imd}}$ & $2.938(11)$ & $8.6(10)$ & 7.1(11) & \\
\hline \multirow{9}{*}{ Co(II)-NikR } & $2 \mathrm{Co}-\mathrm{N}_{\text {imd }}$ & $2.035(15)$ & $3.4(10)$ & $6.1(8)$ & 0.99 \\
\hline & $5 \mathrm{Co}-\mathrm{N}$ & $2.131(5)$ & [3.4] & {$[6.1]$} & \\
\hline & $4 \mathrm{Co}-\mathrm{C}_{\mathrm{imd}}$ & $2.951(11)$ & $5.2(9)$ & $10.0(12)$ & \\
\hline & $3 \mathrm{Co}-\mathrm{N}_{\text {imd }}$ & $2.033(7)$ & $2.8(9)$ & $5.6(7)$ & \multirow[t]{3}{*}{0.96} \\
\hline & $4 \mathrm{Co}-\mathrm{N}$ & $2.149(5)$ & [2.8] & {$[5.6]$} & \\
\hline & $6 \mathrm{Co}-\mathrm{C}_{\mathrm{imd}}$ & $2.957(10)$ & $5.9(9)$ & $10.3(10)$ & \\
\hline & $4 \mathrm{Co}-\mathrm{N}_{\text {imd }}$ & $2.065(5)$ & $3.8(9)$ & $7.2(7)$ & \multirow[t]{3}{*}{0.99} \\
\hline & $3 \mathrm{Co}-\mathrm{N}$ & $2.174(10$ & [3.8] & [7.2] & \\
\hline & $8 \mathrm{Co}-\mathrm{C}_{\mathrm{imd}}$ & $2.950(11)$ & $8.3(9)$ & $8.6(10)$ & \\
\hline
\end{tabular}




\begin{tabular}{|c|c|c|c|c|c|}
\hline \multirow[t]{5}{*}{ Ni(II)-NikR } & $3 \mathrm{Ni}-\mathrm{N}_{\mathrm{imd}}$ & $1.905(3)$ & $1.9(3)$ & $11.8(5)$ & 0.84 \\
\hline & $1 \mathrm{Ni}-\mathrm{S}$ & $2.130(6)$ & $5.3(3)$ & $-16.0(18)$ & \\
\hline & $2 \mathrm{Ni}-\mathrm{N}_{\mathrm{imd}}$ & $1.892(5)$ & $1.3(3)$ & $8.8(6)$ & 0.83 \\
\hline & $1-\mathrm{Ni}-\mathrm{N}$ & $2.060(17)$ & [1.3] & [8.8] & \\
\hline & $1 \mathrm{Ni}-\mathrm{S}$ & $2.184(12)$ & $5.1(9)$ & $11.8(2)$ & \\
\hline \multirow[t]{8}{*}{$\mathrm{Cu}(\mathrm{II})-\mathrm{NikR}$} & $2 \mathrm{Cu}-\mathrm{N}_{\mathrm{imd}}$ & $1.928(8)$ & $4.3(6)$ & $5.1(10)$ & 0.77 \\
\hline & $1 \mathrm{Cu}-\mathrm{N}$ & $2.100(28)$ & {$[4.3]$} & {$[5.1]$} & \\
\hline & $1 \mathrm{Cu}-\mathrm{S}$ & $2.211(6)$ & 4.3(9) & $8.1(16)$ & \\
\hline & $1 \mathrm{Cu}-\mathrm{N}_{\mathrm{imd}}$ & $1.912(26)$ & $8.0(15)$ & $4.8(13)$ & 0.85 \\
\hline & $2 \mathrm{Cu}-\mathrm{N}$ & $1.983(15)$ & [8.0] & {$[4.8]$} & \\
\hline & $1 \mathrm{Cu}-\mathrm{S}$ & $2.237(9)$ & $5.7(7)$ & $12.0(16)$ & \\
\hline & $3 \mathrm{Cu}-\mathrm{N}_{\mathrm{imd}}$ & $1.958(8)$ & $9.7(7)$ & $5.8(9)$ & 0.81 \\
\hline & $1 \mathrm{Cu}-\mathrm{S}$ & $2.236(8)$ & $5.5(6)$ & $11.8(14)$ & \\
\hline \multirow[t]{5}{*}{$\mathrm{Cu}(\mathrm{I})-\mathrm{NikR}$} & $2 \mathrm{Cu}-\mathrm{N}_{\mathrm{imd}}$ & $2.083(11)$ & $5.6(10)$ & $11.2(10)$ & 1.10 \\
\hline & $1 \mathrm{Cu}-\mathrm{S}$ & $2.279(4)$ & $0.2(2)$ & $8.4(13)$ & \\
\hline & $1 \mathrm{Cu}-\mathrm{N}_{\mathrm{imd}}$ & $2.130(16)$ & $2.4(13)$ & $12.5(11)$ & 1.06 \\
\hline & $1 \mathrm{Cu}-\mathrm{N}$ & $2.037(14)$ & [2.4] & [12.5] & \\
\hline & $1 \mathrm{Cu}-\mathrm{S}$ & $2.279(5)$ & $0.2(0.2)$ & $7.9(14)$ & \\
\hline
\end{tabular}




$\begin{array}{llllll}\mathrm{Zn}(\mathrm{II})-\mathrm{NikR} & 3 \mathrm{Zn}-\mathrm{N}_{\text {imd }} & 1.990(6) & 4.9(5) & 2.9(7) & 1.01 \\ 1 \mathrm{Zn}-\mathrm{S} & 2.229(8) & 2.7(5) & 1.3(22) & \\ & & & & & \\ & 3{\mathrm{Zn}-\mathrm{N}_{\text {imd }}} & 1.993(10) & 6.3(7) & 3.3(7) & 0.96 \\ 1 \mathrm{Zn}-\mathrm{N} & 2.040(32) & {[6.3]} & {[3.3]} & \\ 1 \mathrm{Zn}-\mathrm{S} & 2.264(10) & 4.5(7) & 9.9(21) & \end{array}$


Table 12. Unfiltered Fits for Bi-Metallic NikR Complexes:

\begin{tabular}{|c|c|c|c|c|c|}
\hline & $\mathrm{N}$ & $r(\AA)$ & $\sigma^{2}\left(x 10^{3} \AA^{-2}\right)$ & $\Delta \mathrm{E}_{0}$ & GOF \\
\hline CuNi-NikR & $2 \mathrm{Cu}-\mathrm{N}_{\mathrm{imd}}$ & $1.970(10)$ & 7.9(7) & $7.4(7)$ & 0.56 \\
\hline \multirow[t]{5}{*}{$\mathrm{Cu}$-edge } & $1 \mathrm{Cu}-\mathrm{N} / \mathrm{O}$ & $2.018(22)$ & [7.9] & {$[7.4]$} & \\
\hline & $1 \mathrm{Cu}-\mathrm{S}$ & $2.167(16)$ & $16.6(25)$ & $12.5(17)$ & \\
\hline & $1 \mathrm{Cu}-\mathrm{N}_{\text {imd }}$ & $1.943(15)$ & $6.5(9)$ & $6.8(7)$ & 0.56 \\
\hline & $2 \mathrm{Cu}-\mathrm{N} / \mathrm{O}$ & 2.004(9) & [6.5] & [6.8] & \\
\hline & $1 \mathrm{Cu}-\mathrm{S}$ & $2.149(19)$ & $18.3(31)$ & 11.2(19) & \\
\hline CuNi-NikR & $2 \mathrm{Ni}-\mathrm{N}_{\mathrm{imd}}$ & $1.992(6)$ & $3.4(5)$ & $7.8(4)$ & 0.82 \\
\hline \multirow[t]{10}{*}{ Ni-edge } & $4 \mathrm{Ni}-\mathrm{N} / \mathrm{O}$ & $2.106(3)$ & {$[3.4]$} & {$[7.8]$} & \\
\hline & $2 \mathrm{Ni}-\mathrm{N}_{\mathrm{imd}}$ & $1.999(8)$ & $4.2(10)$ & $9.1(6)$ & 0.81 \\
\hline & $3 \mathrm{Ni}-\mathrm{N} / \mathrm{O}$ & $2.149(17)$ & [4.2] & [9.1] & \\
\hline & $1 \mathrm{Ni}-\mathrm{S} / \mathrm{Cl}$ & $2.163(10)$ & $3.1(14)$ & $-11.6(37)$ & \\
\hline & $1 \mathrm{Ni}-\mathrm{N}_{\mathrm{imd}}$ & $1.997(15)$ & $3.4(5)$ & $8.5(5)$ & 0.85 \\
\hline & $4 \mathrm{Ni}-\mathrm{N} / \mathrm{O}$ & $2.099(4)$ & {$[3.4]$} & [8.5] & \\
\hline & $1 \mathrm{Ni}-\mathrm{S} / \mathrm{Cl}$ & $1.995(61)$ & $14.2(3)$ & $-30.6(130)$ & \\
\hline & $3 \mathrm{Ni}-\mathrm{N}_{\mathrm{imd}}$ & $2.035(7)$ & $4.6(8)$ & $10.5(6)$ & 0.81 \\
\hline & $2 \mathrm{Ni}-\mathrm{N} / \mathrm{O}$ & $2.184(50)$ & [4.6] & [10.5] & \\
\hline & $1 \mathrm{Ni}-\mathrm{S} / \mathrm{Cl}$ & $2.197(13)$ & $2.8(12)$ & $-13.9(47)$ & \\
\hline
\end{tabular}




\begin{tabular}{|c|c|c|c|c|c|}
\hline NiCo-NikR in & $2 \mathrm{Ni}-\mathrm{N}_{\mathrm{imd}}$ & $1.938(5)$ & $4.6(6)$ & $11.6(6)$ & 0.62 \\
\hline TrisCl and $\mathrm{NaCl}$ & $1 \mathrm{Ni}-\mathrm{N} / \mathrm{O}$ & $2.321(16)$ & {$[4.6]$} & [11.6] & \\
\hline \multirow[t]{4}{*}{ Ni-edge } & $1 \mathrm{Ni}-\mathrm{S}$ & $2.190(5)$ & $2.8(5)$ & $-4.7(15)$ & \\
\hline & $1 \mathrm{Ni}-\mathrm{N}_{\mathrm{imd}}$ & $1.928(6)$ & $0.4(5)$ & $10.3(7)$ & 0.65 \\
\hline & $2 \mathrm{Ni}-\mathrm{N} / \mathrm{O}$ & $2.086(7)$ & {$[0.4]$} & {$[10.3]$} & \\
\hline & $1 \mathrm{Ni}-\mathrm{S}$ & $2.108(17)$ & $7.8(13)$ & $8.2(18)$ & \\
\hline NiCo-NikR in & $2 \mathrm{Co}-\mathrm{N}_{\mathrm{imd}}$ & 1.931(11) & $5.6(12)$ & $-5.7(12)$ & 0.78 \\
\hline TrisCl and $\mathrm{NaCl}$ & $3 \mathrm{Co}-\mathrm{N} / \mathrm{O}$ & $2.142(28)$ & [5.6] & {$[-5.7]$} & \\
\hline \multirow[t]{10}{*}{ Co-edge } & $1 \mathrm{Co}-\mathrm{S} / \mathrm{Cl}$ & $2.160(20)$ & $6.7(44)$ & $-13.4(39)$ & \\
\hline & $6 \mathrm{Co}-\mathrm{C}_{\mathrm{imd}}$ & $3.121(15)$ & $7.8(15)$ & $15.0(11)$ & \\
\hline & $1 \mathrm{Co}-\mathrm{N}_{\mathrm{imd}}$ & $1.902(52)$ & $9.4(24)$ & $-7.2(10)$ & 0.77 \\
\hline & $4 \mathrm{Co}-\mathrm{N} / \mathrm{O}$ & $1.982(13)$ & {$[9.4]$} & {$[-7.2]$} & \\
\hline & $1 \mathrm{Co}-\mathrm{S} / \mathrm{Cl}$ & $2.201(12)$ & $3.3(10)$ & $-32.8(43)$ & \\
\hline & $2 \mathrm{Co}-\mathrm{C}_{\mathrm{imd}}$ & $3.123(12)$ & $0.6(9)$ & $14.3(17)$ & \\
\hline & $3 \mathrm{Co}-\mathrm{N}_{\mathrm{imd}}$ & $1.949(14)$ & $7.0(10)$ & $-6.3(13)$ & 0.79 \\
\hline & $2 \mathrm{Co}-\mathrm{N} / \mathrm{O}$ & $2.202(36)$ & {$[7.0]$} & {$[-6.3]$} & \\
\hline & $1 \mathrm{Co}-\mathrm{S} / \mathrm{Cl}$ & $2.210(20)$ & $3.4(14)$ & $-15.8(36)$ & \\
\hline & 6 Co- $\mathrm{C}_{\text {imd }}$ & $3.118(14)$ & $11.8(17)$ & $14.6(9)$ & \\
\hline NiCo-NikR in & $2 \mathrm{Ni}-\mathrm{N}_{\mathrm{imd}}$ & $1.884(3)$ & $0.8(2)$ & $9.6(5)$ & 0.65 \\
\hline TrisBr and $\mathrm{NaBr}$ & $1 \mathrm{Ni}-\mathrm{N} / \mathrm{O}$ & $2.100(23)$ & {$[0.8]$} & {$[9.6]$} & \\
\hline Ni-edge & $1 \mathrm{Ni}-\mathrm{S}$ & $2.155(5)$ & $2.4(12)$ & $7.0(13)$ & \\
\hline
\end{tabular}




$\begin{array}{lllll}3 \mathrm{Ni}-\mathrm{N}_{\text {imd }} & 1.897(2) & 2.7(2) & 11.2(3) & 0.59 \\ 1 \mathrm{Ni}-\mathrm{N} / \mathrm{O} & 2.343(10) & {[2.7]} & {[11.2]} & \\ 1 \mathrm{Ni}-\mathrm{S} & 2.159(6) & 1.6(2) & -8.1(16) & \end{array}$

\begin{tabular}{|c|c|c|c|c|c|}
\hline NiCo-NikR in & $2 \mathrm{Co}-\mathrm{N}_{\mathrm{imd}}$ & $1.926(9)$ & $2.7(6)$ & $-3.0(9)$ & 0.94 \\
\hline TrisBr and $\mathrm{NaBr}$ & $3 \mathrm{Co}-\mathrm{N} / \mathrm{O}$ & $2.084(7)$ & {$[2.7]$} & {$[-3.0]$} & \\
\hline \multirow[t]{7}{*}{ Co-edge } & $4 \mathrm{Co}-\mathrm{C}_{\mathrm{imd}}$ & $3.081(12)$ & $3.8(9)$ & $0.6(14)$ & \\
\hline & $1 \mathrm{Co}-\mathrm{N}_{\mathrm{imd}}$ & $1.905(28)$ & $7.6(11)$ & $-3.1(12)$ & 1.08 \\
\hline & $4 \mathrm{Co}-\mathrm{N} / \mathrm{O}$ & $2.055(9)$ & {$[7.6]$} & {$[-3.1]$} & \\
\hline & $2 \mathrm{Co}-\mathrm{C}_{\mathrm{imd}}$ & $3.626(32)$ & $4.6(34)$ & $18.3(14)$ & \\
\hline & $3 \mathrm{Co}-\mathrm{N}_{\mathrm{imd}}$ & $2.009(9)$ & $8.1(21)$ & $1.1(8)$ & 1.06 \\
\hline & $2 \mathrm{Co}-\mathrm{N} / \mathrm{O}$ & $2.127(26)$ & [8.1] & {$[1.1]$} & \\
\hline & $6 \mathrm{Co}-\mathrm{C}_{\mathrm{imd}}$ & $3.043(14)$ & $6.6(11)$ & $-4.0(14)$ & \\
\hline
\end{tabular}



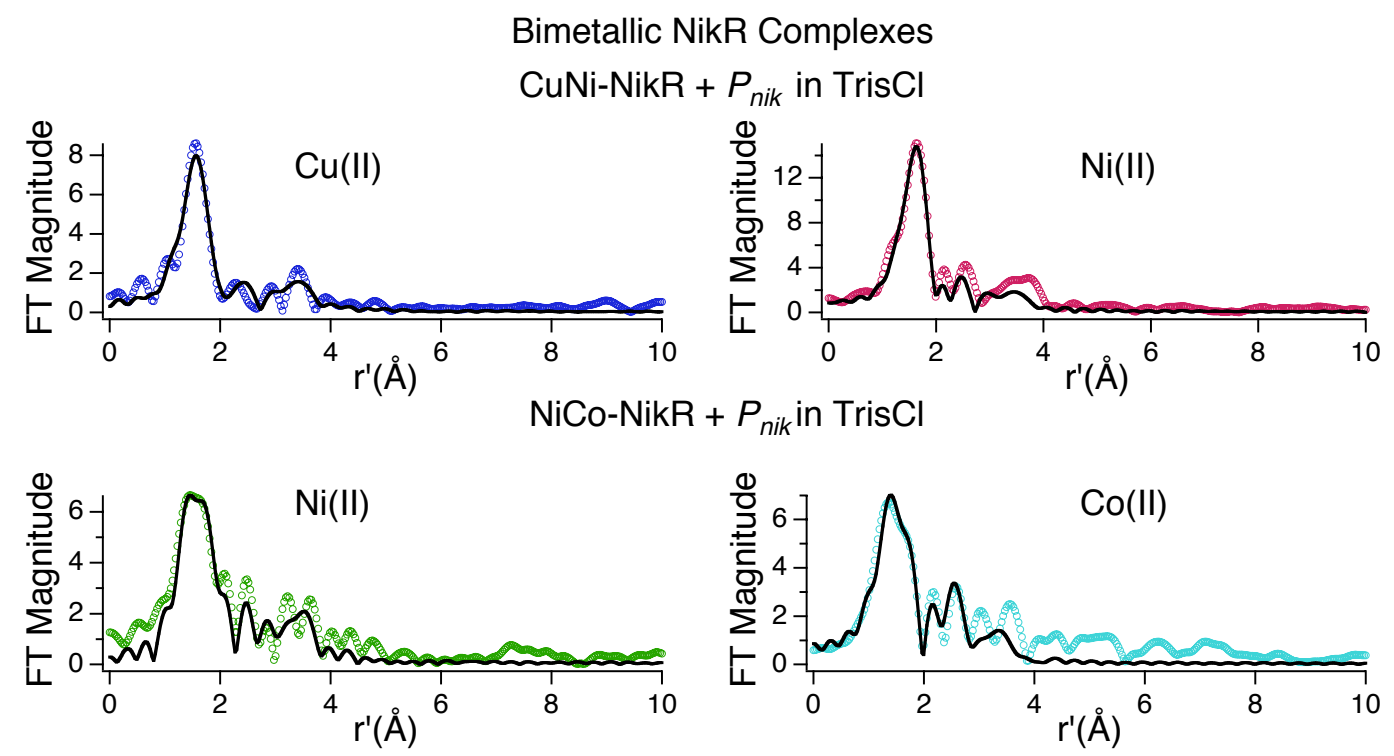

$\mathrm{NiCo}-\mathrm{NikR}+P_{\text {nik }}$ in TrisBr
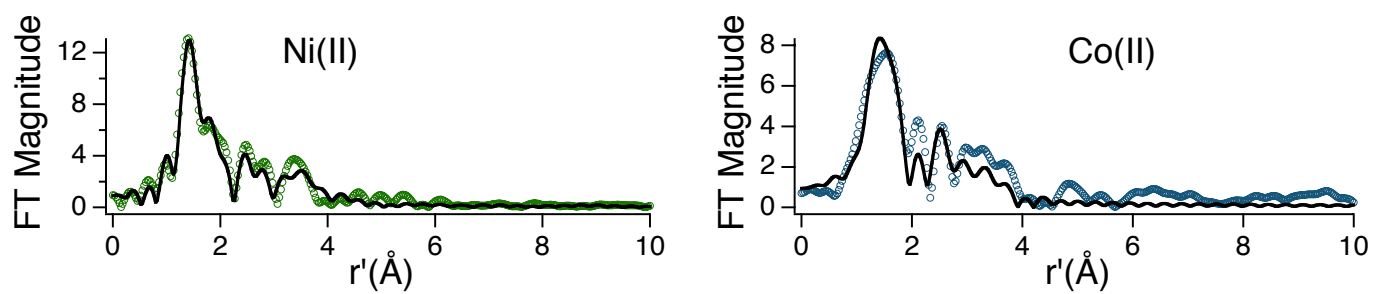

Figure 1. Fourier-transform spectra for the high-affinity metal binding site (left) and the low-affinity metal binding site (right). 


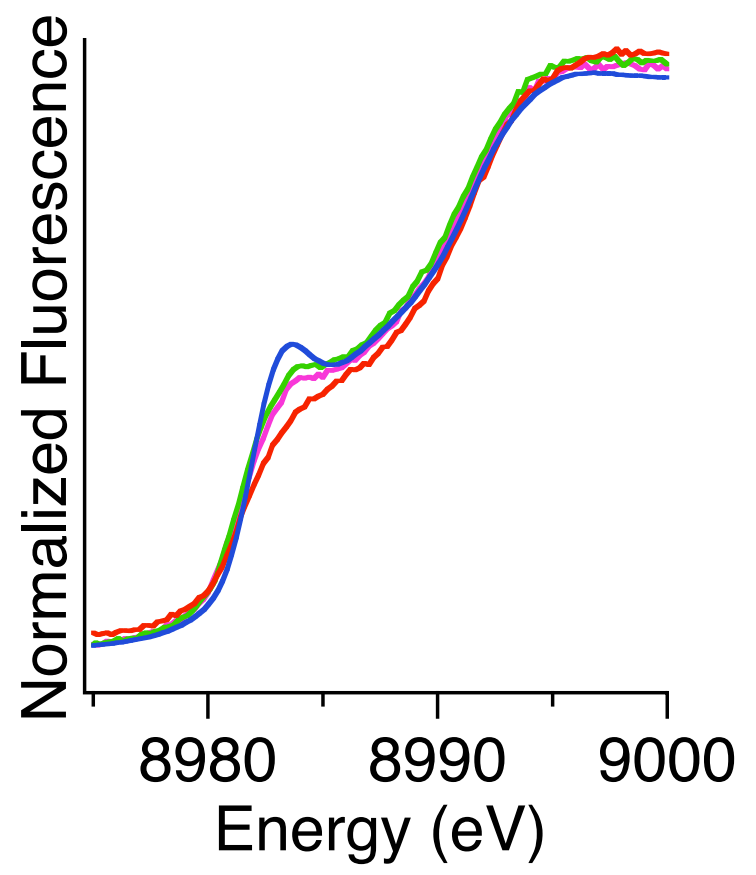

Figure 2. Evidence of photoreduction due to $\mathrm{x}$-ray exposure of the $\mathrm{Cu}$ site in $\mathrm{CuNi}$ NikR. Red, pink and green are $\mathrm{Cu} K$-edge scans 1,2 , and 3 respectively of CuNi-NikR. Blue is a $\mathrm{Cu} K$-edge data post collection of Ni $K$-edge data of CuNi-NikR.

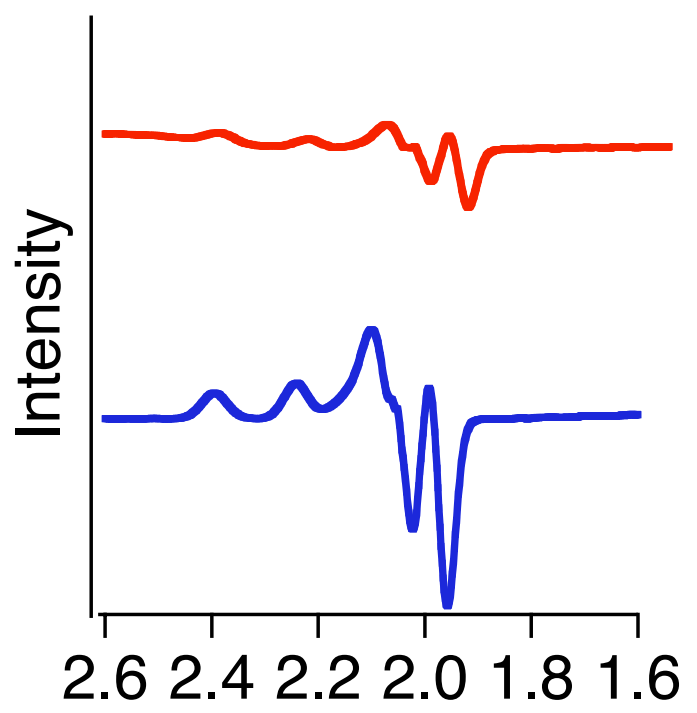

Figure 3. X-Band EPR spectra of Cu(II)-NikR (blue) and CuNi-NikR (red). 


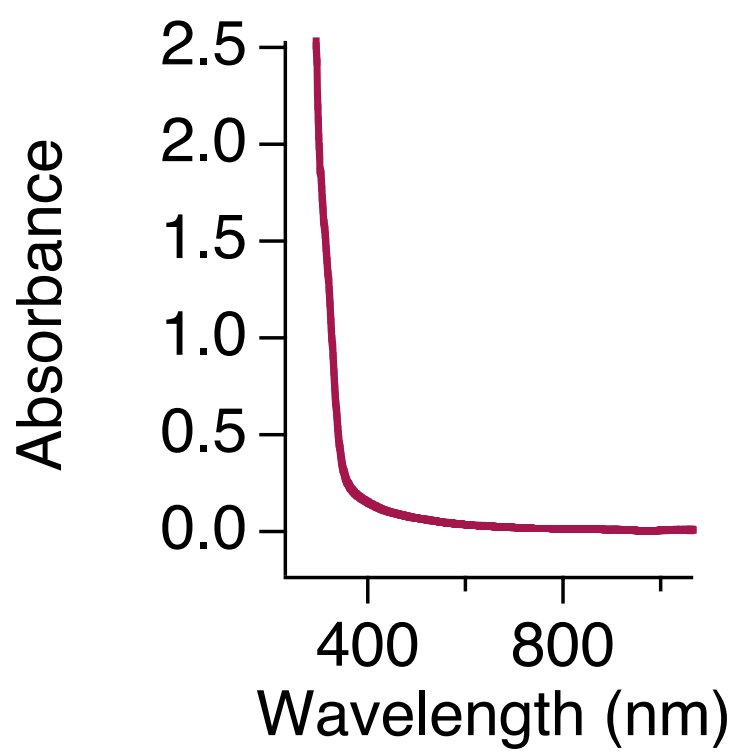

Figure 4. UV-vis spectrum of $\sim 0.4 \mathrm{mM} \mathrm{Co(II)-NikR} \mathrm{in} 20 \mathrm{mM}$ Tris (pH 8.3 ), $100 \mathrm{mM}$ $\mathrm{NaCl}$. 

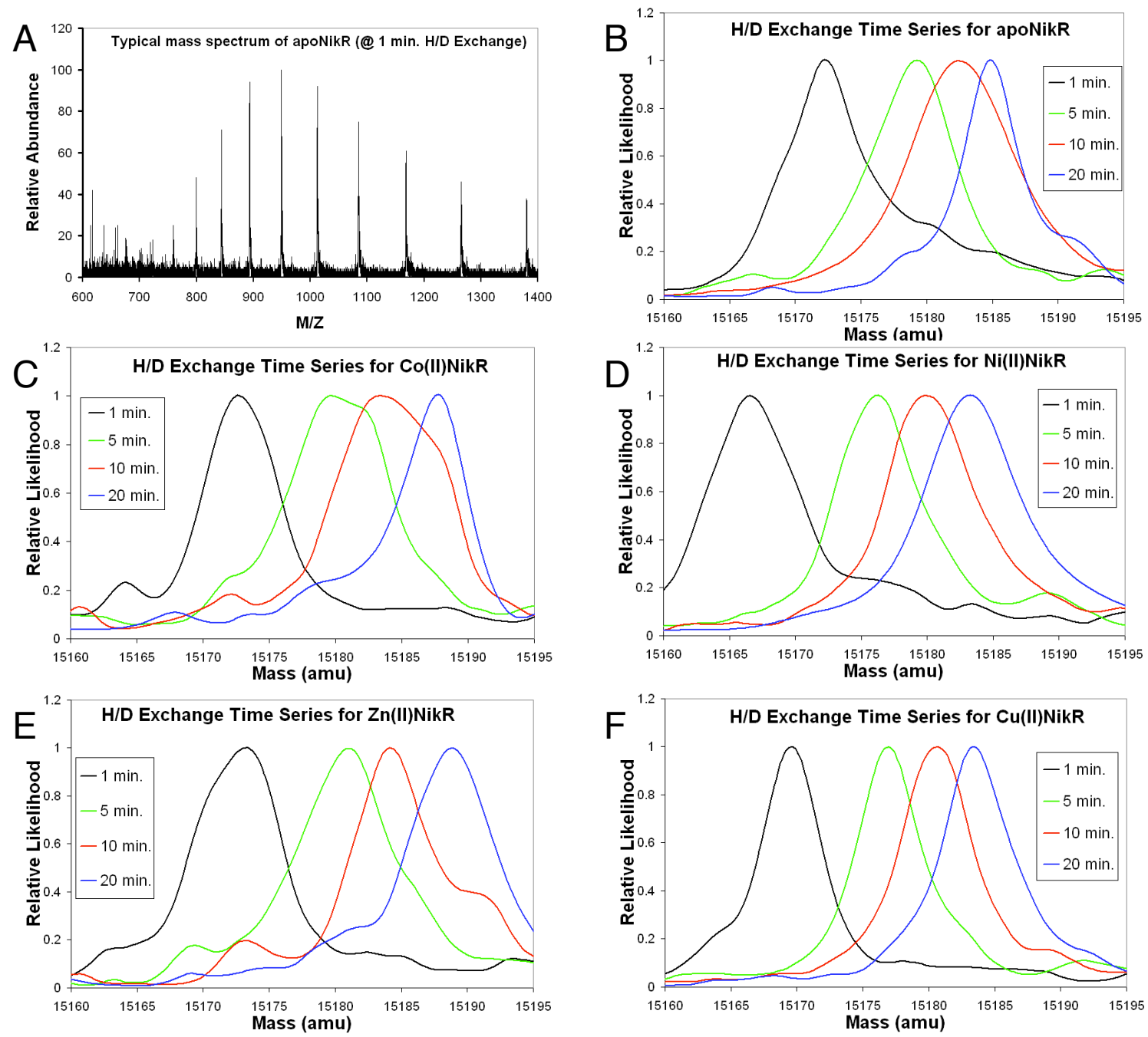

Figure 5. Mass spectrometry data used for the analysis of hydrogen/deuterium exchange experiments. Panel A, representative mass/charge spectrum for NikR (apo NikR after 1 nimute of H/D exchange). The peaks between $\sim 723$ and $1400 \mathrm{~m} / \mathrm{z}$ represent the charge distribution $(+21$ to +11$)$ seen for a NikR monomer under the electrospray ionization conditions used here (80\% methanol, $19.7 \%$ water, $0.3 \%$ TFA, v/v/v, $\mathrm{pH} \sim 2.5$ ). Peaks lower than $700 \mathrm{~m} / \mathrm{z}$ are most likely non-protein molecules. Mass/charge spectra as in panal A were deconvoluted for each H/D exchange time point using the MaxEnt1 algorithm from MassLynx 4.0 for the mass range 14000 - 16000 Da (undeuterated NikR 
monomer mass was $15093.93 \mathrm{Da}$ ). The deconvoluted data for a single series of H/D exchange time points for apo-NikR is shown in panel B and each metal-substituted NikR are shown individually in Pannels C-F. For each curve, the mass value at the peak maximum was used for determining the averages and standard deviations of the deuterium uptake difference relative to apo-NikR. These data were collected on the same day and are representative of the three independent time point series used for the difference data (apo - metal bound) shown in Figure 3. 


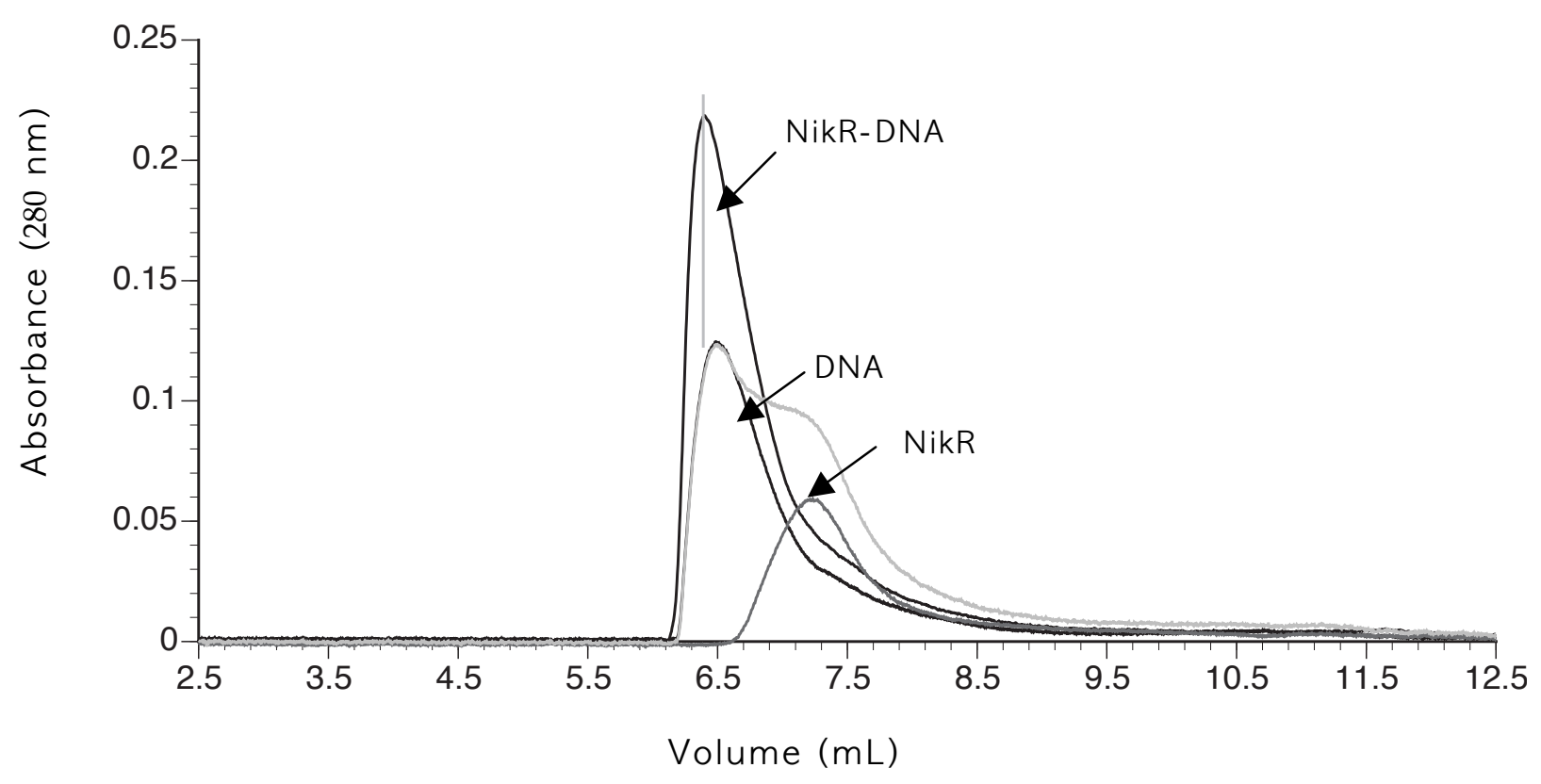

Figure 6. The NikR-DNA complex is stable under gel-filtration conditions. A BioSelect SEC125-5 column (300 mm x $7.8 \mathrm{~mm} ; 15 \mathrm{~mL}$ volume; Bio-Rad) was equilibrated with $20 \mathrm{mM}$ Tris (pH 7.5), $200 \mathrm{mM} \mathrm{NaCl}, 3 \mathrm{mM} \mathrm{MgCl} 2,100 \mathrm{nM} \mathrm{NiCl}{ }_{2}$ (flow-rate 1 $\mathrm{mL} / \mathrm{min}$ ). A NikR-DNA sample was incubated in the equilibration buffer for $15 \mathrm{~min}$ before loading ( $1 \mathrm{mM} \mathrm{NikR}$ and $22 \mu \mathrm{M}$ DNA, total volume $400 \mu \mathrm{L})$. NikR and DNA only controls were loaded onto the column at the same concentrations used in the NikRDNA sample. The elution volumes of the absorbance maxima of each sample were: NikR-DNA, $6.23 \mathrm{~mL}$; DNA, $6.28 \mathrm{~mL}$; NikR $7.18 \mathrm{~mL}$. The NikR and DNA only traces were superimposed (gray dashes) to show the expected chromatographic profile if the protein-DNA complex did not form. The vertical gray line highlights the offset between the elution maxima of NikR-DNA and DNA only. 